\title{
Design of Passive RFID Sensor Tags Enhanced by a Novel Logical Communication Procedure over LLRP
}

\author{
Luca Catarinucci, Riccardo Colella, and Luciano Tarricone
}

\begin{abstract}
Over the past decade, electromagnetic and communication science societies, along with improving the classical RFID technology, have put in a great deal of effort in designing novel and more complex UHF RFID tags with augmented capabilities. Novel tags offer additional functionalities besides identification by embedding sensors, actuators, and processing units. In this work an enhanced version of one of such devices, called SPARTACUS, is presented. While being completely passive, it conjugates identification, sensing, local computing, and actuation control and enables a proactive communication with any standard RFID reader. The paper presents details on a novel logical communication procedure over Low Level Reader Protocol (LLRP), besides discussing system validation and performance evaluation.
\end{abstract}

Index Terms - Internet of Things, Sensors, LLRP, Power Management, RFID augmented Tags, UHF, Antennas.

\section{INTRODUCTION}

Sustained by the technological advances in Radiofrequency Identification (RFID), new RFID-based devices with augmented capabilities have appeared in recent years in the literature [1, 10]. Some of them, including the one recently published by the same authors and named SPARTACUS (Self-Powered Augmented RFID Tag for Autonomous Computing and Ubiquitous Sensing) [9, 10], conjugate canonical RFID identification with extra functionalities such as sensing, computation, data storing, and actuation. As deeply clarified in [9], this augmented tag embeds an RF power management block which exploits the electromagnetic energy emitted by the RFID reader to power up a microcontroller (MCU), a memory bank, sensors, actuators and alert devices.

In this paper, a new version of SPARTACUS provided with a new physical layer implementing a logical communication procedure over Low Level Reader Protocol (LLRP) is presented as an extension of the paper presented at Splitech 2016 conference [10].

The device is conceived to enable challenging Internet of

Manuscript received January 20, 2017; revised May 16, 2017. Date of publication June 1, 2017.

Authors are with the Department of Innovation Engineering of University of Salento, 73100 Lecce, Italy (e-mails: \{luca.catarinucci, riccardo.colella, luciano.tarricone \}@unisalento.it).

Digital Object Identifier (DOI): 10.24138/jcomss.v13i2.380
Things (IoT) applications, since besides sensing, it provides self-powering, self-computing, self-reasoning, RFID Gen2 compliance, alerting, and long working ranges. Moreover, it supports proactive communication with the reader by implementing a sort of "cooperative reasoning", based on both context information, known at the reader stage, and local data, measured by the tag or stored in its memory.

From implementation point of view, SPARTACUS is equipped with two specifically designed antennas with orthogonal polarization, one feeding the RF power management system and the other the RFID communication system. To preserve energy efficiency, the antennas and each RF section have been carefully designed taking into account mutual interaction between the two RF frontends.

Differently from [10] (where the suitability of SPARTACUS in a building automation context is demonstrated), the novel generalized LLRP-based communication procedure proposed in this work makes tag and reader collaborate together to solve a generic problem based on the local knowledge of the tag and the global knowledge of the reader. Data format, timing and commands have been now defined through an upgrade of the firmware making the tag suitable for general purpose applications.

The device has been exhaustively tested and validated in terms of both electromagnetic characterization and working principle. The achieved results demonstrate that the improved device is a promising solution for implementing a real autonomous and distributed RFID-based passive computing in IoT scenarios.

\section{Augmented TAg Design}

In order to explain the structure of the system, a block diagram illustrating the SPARTACUS general architecture is reported in Fig. 1. As known [9], it is subdivided into three functional blocks. Two of them, representing the SPARTACUS core, are dedicated to the RF power management and the generation and management of the digital information. The third one is the antenna block, consisting of a system of two antennas designed for feeding the power management block and the communication block, respectively. In this way, when the device is in the region covered by an RFID reader, the system is fed through the 


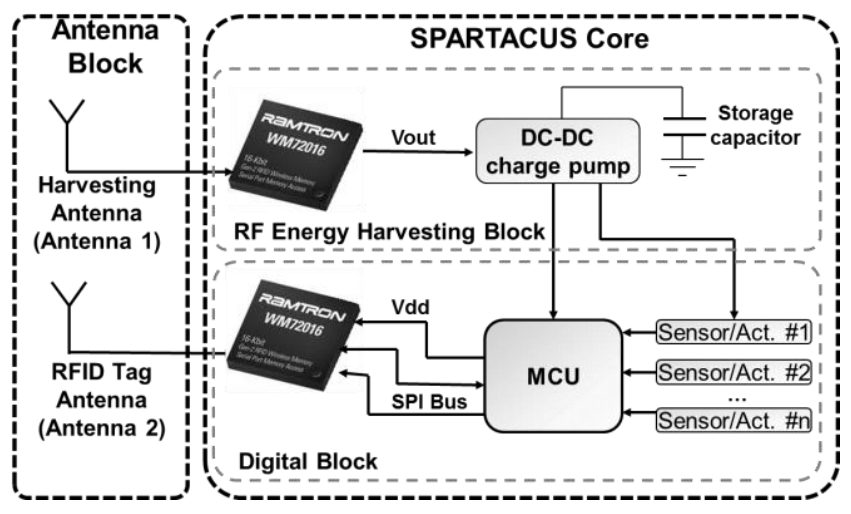

Fig. 1. SPARTACUS Block Diagram [9].

appropriate antenna, thus providing the digital block with the power necessary to operate and to communicate with the reader by means of the other antenna. As can be observed in Fig. 1, a specific capacitor, whose value must be set on the basis on the specific application, has been also foreseen for storing the energy.

Regarding the implementation, a prototype of the system has been realized on $800 \mu$ m-thick FR4 board $\left(\varepsilon_{\mathrm{r}}=4.7\right)$. In particular, the digital block is based on a Microchip ultra-low power 16-bit MCU model PIC24F16KA102 (maximum absorbed current $1.3 \mathrm{~mA}$ ), programmed to control both an analog temperature sensor Texas Instruments model TMP20 (maximum current consumption $4 \mu \mathrm{A}$ ) and an analog light sensor based on a photo-resistor (maximum current consumption $20 \mu \mathrm{A}$ ). Both of them are controlled through the MCU-embedded 10-bit ADC. Moreover, the circuit is provided with an LED for alerting purposes and with a 6-pin connector allowing the control of up to two extra external actuators or sensors [9]. The RFID-based data transmission is in charge of a WM72016 RFID chip communicating with the MCU by means of the SPI interface [11].

An important electromagnetic issue has been the careful design of the two antennas since neither frequency nor spatial diversity techniques could be adopted in the specific case. Nevertheless, taking advantage of the circularly polarized signal emitted by generic RFID reader antennas, polarization diversity can be successfully exploited. Indeed, the RF frontend of SPARTACUS is basically composed of two meandered dipolelike cross-polarized antennas and a common loop between them. In this way, each of the two antennas is composed of a loop and a half wavelength meandered dipole, which implement the socalled T-Match strategy for RFID tag design [12]. Fig. 2a illustrates the layout of the proposed antenna system, whilst the final prototype of SPARTACUS is shown in Fig. $2 b$.

\section{NOVEL SPARTACUS-READER COMMUNICATION PROCEDURE}

In this Section, the logical communication procedure over Low Level Reader Protocol (LLRP) exploited by SPARTACUS to communicate with the reader in a bi-directional manner is presented. More in details the proposed approach makes tag and reader collaborate together to solve a common problem based on the local knowledge of the tag and the global knowledge of the reader through a cooperative interaction. Fig. 3 shows the
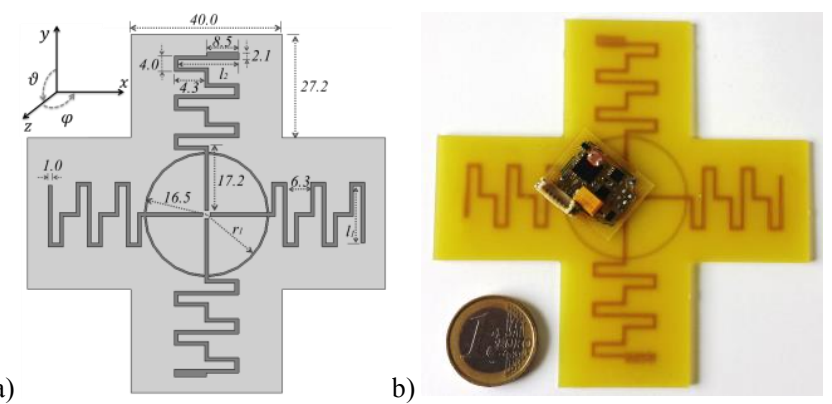

Fig. 2. Layout of the SPARTACUS antenna block (Dimensions are in millimeters) (a), Photo of the device (b).

communication scheme. As observed, once energized and interrogated by the reader, the tag continuously sends the EPC by performing a bidirectional low-level communication according to gen 2 standard timing. Thus, the tag is identified as long as it is located in the reader antenna radiation area. In the meanwhile the augmented tag performs two different operations in a cyclical and asynchronous way whose timing depends on both the level of available RF energy and the storage capacitor value: 1) it starts charging the capacitor, and 2) it passively computes, manages memory, senses the environment and updates its status.

In particular, let's suppose that SPARTACUS needs data from the reader in order to perform a certain computation. With reference to the path highlighted in Fig. 3, when its MCU is energized, the tag changes certain memory bits to specify the required data. Then, the reader reads the memory through gen2 commands and, on the basis of data availability, updates the memory accordingly. Once these data are accessed by SPARTACUS, its MCU retrieves them

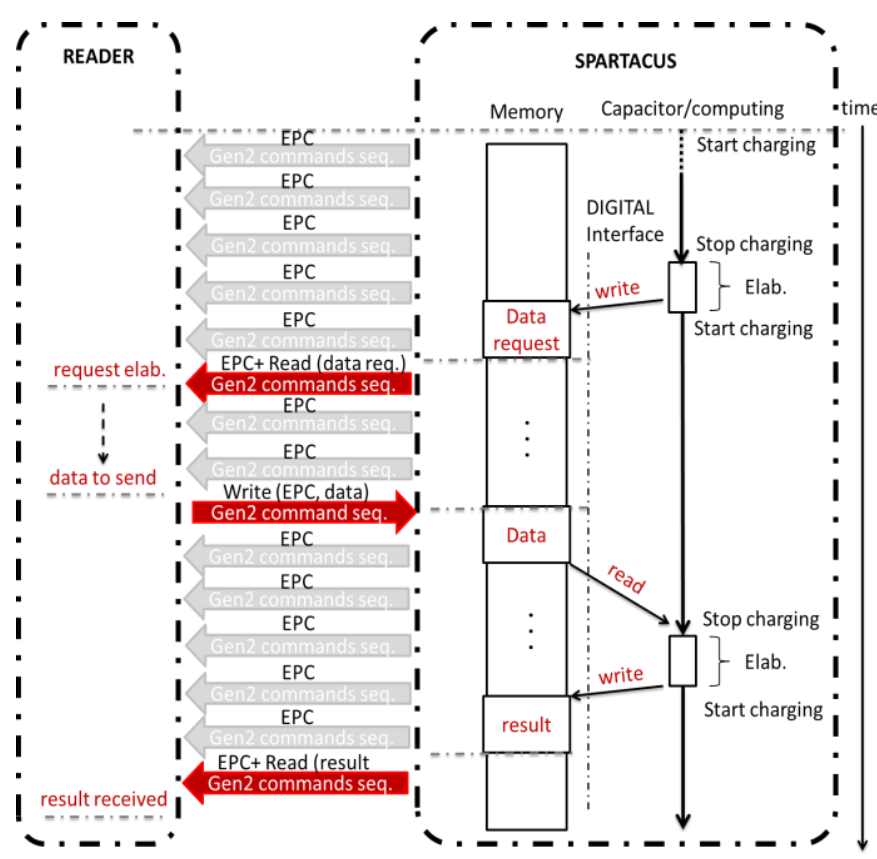

Fig. 3. Example of SPARTACUS-Reader interaction

from the memory at the next energization step, performs the computation, and writes the result into the memory. The reader can now use this information for further elaborations or interactions. 
Table I. Performance By Varying the Capacitor value at 1 meter

\begin{tabular}{llll}
\hline \multicolumn{1}{c}{ Storage Capacitor } & Write Cycles (16-bit word) & $\begin{array}{l}\text { Charging Time [ms] } \\
\text { Idle Mode }\end{array}$ & $\begin{array}{l}\text { Discharging Time [ms] } \\
\text { Run Mode }\end{array}$ \\
\hline $10 \mu \mathrm{F}$ & 4 & 620 & 1.3 \\
\hline $22 \mu \mathrm{F}$ & 8 & 970 & 2.8 \\
\hline $68 \mu \mathrm{F}$ & 24 & 2870 & 8.9 \\
\hline $120 \mu \mathrm{F}$ & 48 & 4820 & 16.2 \\
\hline
\end{tabular}

Finally, a crucial aspect related to this kind of communication is the timing regulating the SPARTACUS-reader interaction. On the one hand, being the device gen2 compliant, the interaction with the reader occurs with the standardized timing specifications, as in the case of a canonical RFID tag. Consequently, when SPARTACUS is in the reader area, it is recognized and can also exchange LLRP commands. On the other hand, the updating of the tag memory done by the device itself is governed by a different timing which depends on many factors: amount of available RF energy, computational load, working distance, reader antennas planning, specific application and, finally, on-board storage capacitor. In particular, this last must be properly set to minimize the computational latency case by case.

\section{RESULTS}

To characterize the new device and validate its functionalities, two different kinds of results will be addressed in this Section. First, the computational performance of the proposed system will be verified. Second, a test involving all the functions of SPARTACUS will be presented.

\section{A. Computational performance and system validation}

As asserted in the previous Section, the computational performance of the device is regulated by a storage capacitor connected to the power management block. Indeed, a high capacitance value allowed a longer processing time at the cost of a much longer recharge time, and a low capacitance value a very fast capacitor recharge, but fewer operations each time. This means that the capacitor should be tuned to establish a good trade-off between charging time and processing time according to the specific application. Table I presents the results obtained by varying the capacitor from $10 \mu \mathrm{F}$ to $220 \mu \mathrm{F}$, along with the capacitor charging and discharging time, corresponding to the idle time and the run time of SPARTACUS, respectively.

As a proof of the concept, and to demonstrate the capabilities of SPARTACUS in sensing environmental events, reasoning, and undertaking actions, a proper test has been performed. On the one hand, SPARTACUS has been equipped with a $10-\mu \mathrm{F}$ capacitor and programmed to turn the LED on when the environmental light level (see Fig. 4a) detected by the local sensor becomes lower than a preset threshold (continuous horizontal line at the bottom of Fig. 4b). On the other hand, a software application controlling the RFID reader has been implemented to process light data transmitted by the device and to send back, on the basis of specific decisions, commands or instructions towards the tag. More specifically, the task of the reader consists in turning the LED off when, and only when, the received light level exceeds a certain threshold (dashed horizontal line at the top of Fig. 4b). The test has been performed by moving SPARTACUS in the reader coverage area, and by varying the ambient light intensity for a total test time of 300 s. Fig. 4b, shows the light values measured by SPARTACUS and received by the reader, whilst Fig. 4c demonstrates that the LED is correctly activated (by SPARTACUS) and deactivated (by the reader), according to preset thresholds. Specifically, continuous spikes represent the LED-activation events locally performed by the tag, whilst dashed spikes are LED-deactivation commands computed by the reader and communicated to the tag. This test clearly demonstrates that SPARTACUS enables selfpowering, self-computation, self-reasoning, sensing, alerting, and distributed computation.

\section{B. Use Case}

In this Section a validation use case concerning the implementation of a SPARTACUS-based system capable to control a fan located in an office room when certain conditions are verified is presented and discussed. In
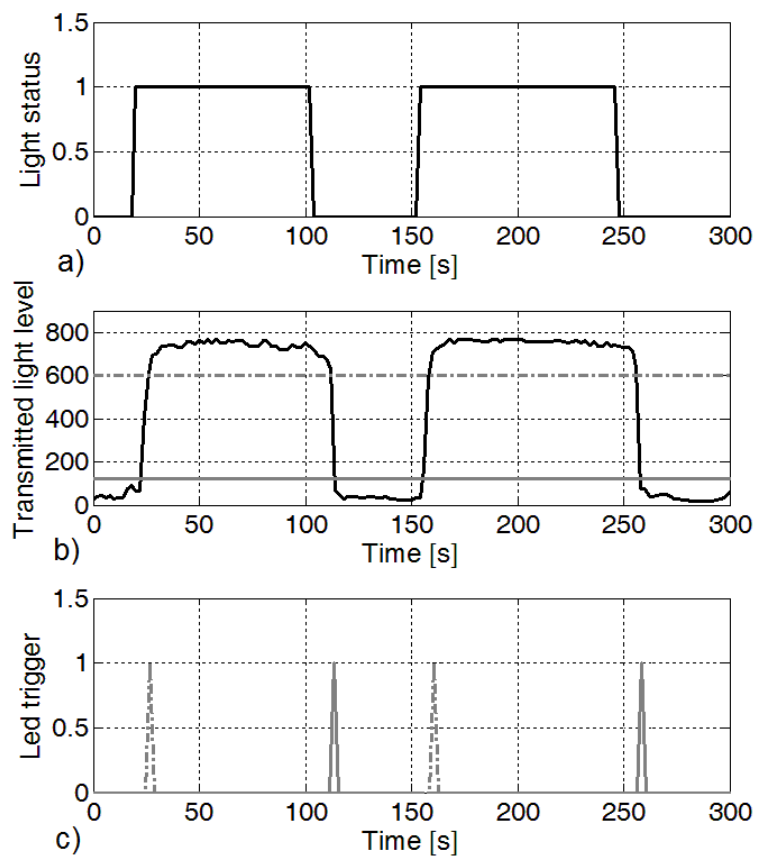

Fig. 4. Light activation/deactivation timeline (a), SPARTACUS transmitted light level (b), SPARTACUS LED trigger generated by SPARTACUS (continuous spikes) and by the reader (dotted spikes) (c).

particular, it is required to switch on the fan only when the following conditions occur: (a) somebody is in the office room, (b) it is daytime, and (c) the external temperature exceeds $28.8^{\circ} \mathrm{C}$. 
In order to detect conditions (a) and (b), a passive infrared sensor (PIR) and an analog light sensor are mounted on SPARTACUS. As for condition (c), a weather station mounted on the roof of our building is periodically interrogated through a computer. The same computer interfaces an Impinj Speedway Revolution RFID reader. Finally, the GPIO of the RFID reader is connected to the fan through an appropriate hardware driver supporting higher power than GPIO. The reader receives temperature values from the laptop and compares them with the $28.8^{\circ} \mathrm{C}$ threshold. Any threshold crossing is communicated to SPARTACUS, when requested. Vice versa, according to the algorithm sketched in Fig. 5, SPARTACUS is programmed to: 1) sense the light level and compare it with a threshold of 2500 Lux (daylight threshold); 2) verify the presence of people in the office room through a PIR sensor; 3) ask the reader about the fan status (ON/OFF); 4) ask the reader whether or not the temperature threshold is exceeded; 5) verify the true/false status of conditions (a), (b), and (c); 6) send to the reader, if needed, a trigger command to switch ON/OFF the fan. The test was performed leaving the device (provided with the default storage capacitor of $10 \mu \mathrm{F}$ ) in a standard office room for 24 hours, 2meter away from the reader antenna, and rather close to a window to monitor the light conditions over the course of a day. Fig. 6 shows how sensor measurements performed by the tag are consistent with events occurred over the 24-hour observation period, with light rising around 6:00 and dropping around 19:30. This clearly demonstrates the ability of SPARTACUS to perform RFID-sensing. Also people presence in the office room, revealed by the PIR sensor and known only by the tag, is visible in Fig. 6, along with external temperature and related threshold, known at the reader stage. It can be observed that the fan is correctly activated/deactivated by the tag according to the occurrence of the mentioned conditions. This test definitively demonstrates the suitability of SPARTACUS to enable novel perspective in frameworks like distributed sensor networks and collaborative mobile crowd sensing $[13,14]$.

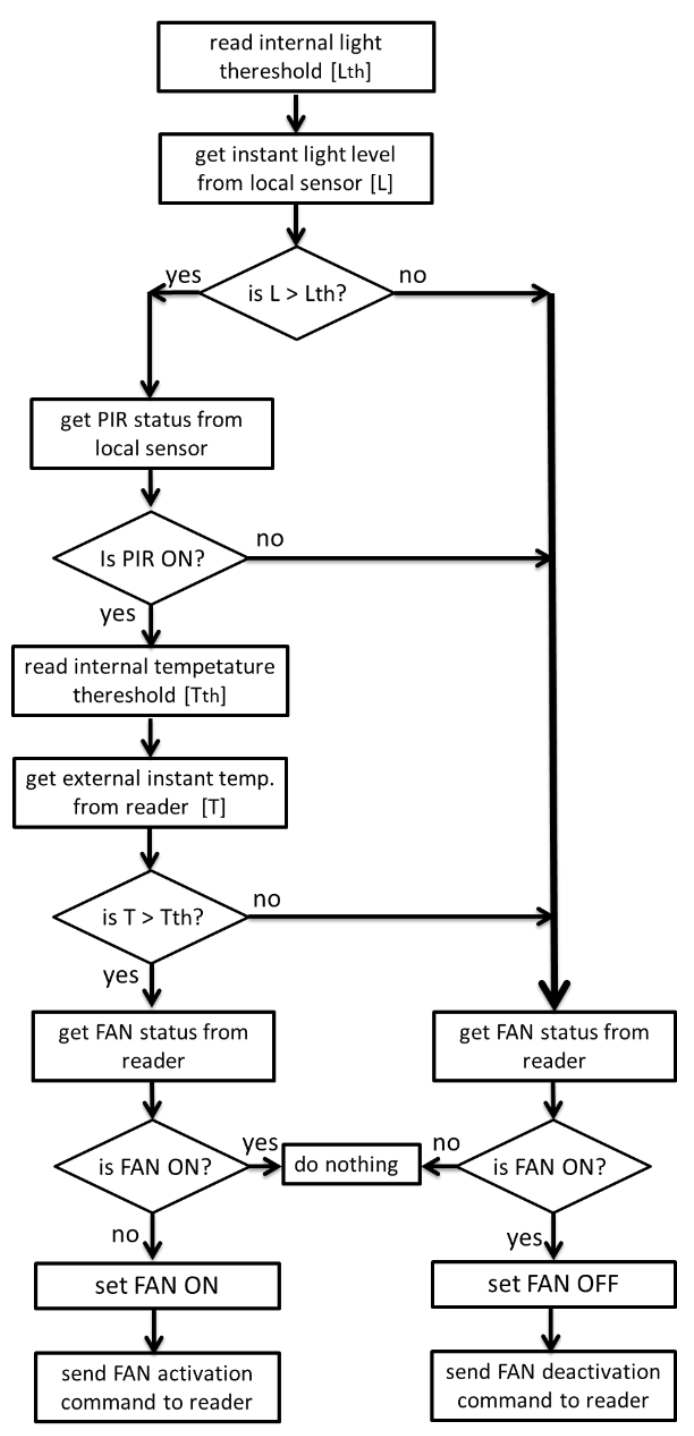

Fig. 5. Algorithm performed by SPARTACUS for the validation test.

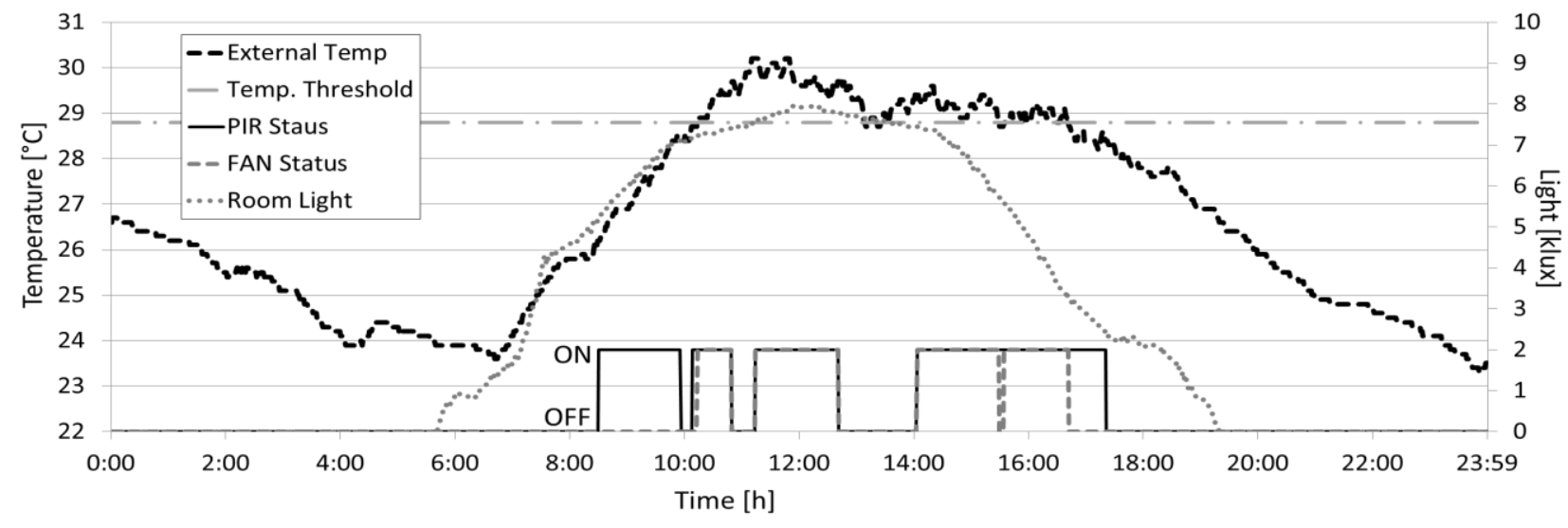

Fig. 6. RFID-based ambient control on the basis of local and context data.

\section{CONCLUSIONS}

In this paper, an improved augmented RFID tag based on

Low Level Reader Protocol enabling a novel tag-reader 
cooperative approach for reasoning and sensing has been presented. Fed by an $866 \mathrm{MHz}$ antenna system composed of two meandered dipoles in cross-configuration and a common loop, the device features an energy management section, which powers up an ultra-low-power microcontroller, a thermometer, an ambient light sensor, and an LED diode. Once designed the system has been implemented and tested in some application contexts which take advantage from several features of the renovated device: the capability of asking for and receiving context data, of sensing a physical value, of reasoning, and of taking decisions, have been exploited at the same time.

\section{REFERENCES}

[1] Timeseries," Information Hiding, LNCS, vol. 3727, pp. 234-246, 2005.

[2] A. P. Sample, D. J. Yeager, P. S. Powledge, A. V. Mamishev, and J. R. Smith, "Design of an RFID-based Battery-Free Programmable Sensing Platform," IEEE Transactions on Instrumentation and Measurement, vol. 57, no. 11, pp. 26082615, Nov. 2008.

[3] R. S. Nair, E. Perret, S. Tedjini, T. Baron, "A Group-DelayBased Chipless RFID Humidity Tag Sensor Using Silicon Nanowires," Antennas and Wireless Prop. Letters, IEEE, vol.12, pp.729-732, 2013.

[4] L. Catarinucci, R. Colella, and L. Tarricone, "Enhanced UHF RFID Sensor-Tag," IEEE Microwave and Wireless Components Letters, vol. 23, no. 1, pp. 49-51, Jan. 2013.

[5] L. Catarinucci, R. Colella, L. Mainetti, L. Patrono, S. Pieretti, I. Sergi, L. Tarricone, "Smart RFID Antenna System for Indoor Tracking and Behavior Analysis of Small Animals in Colony Cages," IEEE Sensors Journal, vol.14, no.4, pp.1198-1206, April 2014.

[6] C. Occhiuzzi, C. Paggi, and G. Marrocco, "Passive RFID StrainSensor Based on Meander-Line Antennas," IEEE Transactions on Antennas and Propagation, vol. 59, no. 12, pp. 4836-4840, Dec. 2011.

[7] A. A. Babar, S. Manzari, L. Sydanheimo, and A. Z. Elsherbeni, "Passive UHF RFID Tag for Heat Sensing Applications," IEEE Transactions on Antennas and Propagation, vol. 60, no. 9, pp. 4056-4064, Sept. 2012.

[8] C. Occhiuzzi, G. Contri, and G. Marrocco, "Design of Implanted RFID Tags for Passive Sensing of Human Body: The STENTag," IEEE Transactions on Antennas and Propagation, vol. 60, no. 7, pp. 3146-3154, July 2012.

[9] R. Colella, L. Tarricone, L. Catarinucci, "SPARTACUS: SelfPowered Augmented RFID Tag for Autonomous Computing and Ubiquitous Sensing," IEEE Transactions on Antennas and Propagation, vol. 63, no. 5, pp. 2272-2281, May 2015.

[10] R. Colella, L. Catarinucci, L. Tarricone, "Passive RFID tag with sensing and reasoning capabilities for building automation," International Multidisciplinary Conference on Computer and Energy Science, SpliTech 2016, Split, Croatia, July 13-15, 2016.

[11] Cypress Semiconductor Corp., Ramtron WM 72016 RFID Chip. [Online]. Available: http://www.cypress.com.

[12] G. Marrocco, "The art of UHF RFID antenna design: Impedance Matching and size reduction techniques," IEEE Antennas and Propagation Magazine, Vol. 50, No. 1, 66-79, 2008.

[13] A. Longo, M. Zappatore, M.A. Bochicchio, "Collaborative learning from Mobile Crowd Sensing: A case study in electromagnetic monitoring," IEEE Global Engineering Education Conference, EDUCON, 2015-April, art. no. 7096052, pp. $742-750$.

[14] A. Longo, M. Zappatore and M.A. Bochicchio, "Towards Massive Open Online Laboratories: an Experience about
Electromagnetic Crowdsensing", 2015 12th Int. Conf. on Remote Engineering and Virtual Instrumentation, REV 2015, Bangkok, Thailand, pp. 43-51, 2015.

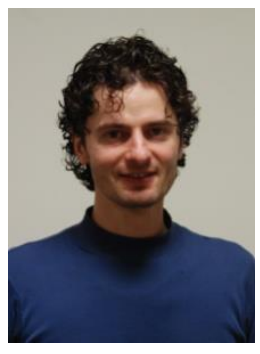

Luca Catarinucci received the Laurea degree (with honors) in Electronic Engineering from the University of Perugia, Italy, in 1998. Since 2003, he has been with the University of Salento, Lecce, Italy, where he is currently an Associate Professor of Electromagnetic Fields and Professor of "Microwaves" and "Electromagnetic Solutions for Hi-Tech" with the Department of Innovation Engineering. He authored more than 150 papers published in international journals and conferences and four chapters books with international diffusion. He holds two patents. His research activity has been mostly focused on the implementation of highperformance electromagnetic simulation tools, on the electromagnetic characterization of heterogeneous materials, on the use of time-domain reflectometry for the qualitative and quantitative characterization of fluids. Currently he is strongly involved in RFID-related activities, ranging from antenna and system design, integration between sensors and RFID tags, RFIDbased robot navigation, and new techniques for tag characterization, optimization, and design.

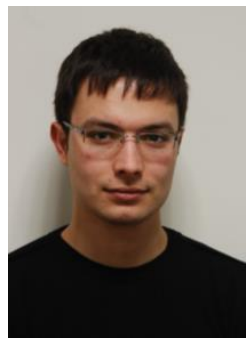

Riccardo Colella received the M.Sc. (with honors) degree in telecommunication engineering from the University of Salento in 2010 and currently he is a Research Fellow in the electromagnetic fields sector of the same University. His research activity is focused on the design and optimization of RFID SensorTags, 3D-printed RFID antennas and their applications in Wireless Sensor Networks. He authored more than 60 papers appeared on international journals and in national and international conferences, 2 chapter books with international diffusion and a Patent. He has been awarded with the prize "Best Thesis on ICT" assigned by CNIT (National Inter-university Consortium on Telecommunications), Confindustria and AICA (Italian Association for Informatics and Automated Computing) in 2011. He has been also awarded with the IEEE MTT-S Central-Southern Italy Award in 2013.

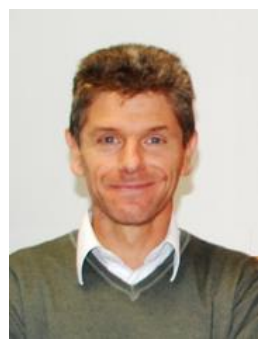

Luciano Tarricone received the Laurea degree (cum-laude) in electronic engineering and the Ph.D. degree from Rome University "La Sapienza," Rome, Italy, in 1989 and 1994, respectively. Since 1994, he has been a Researcher at the University of Perugia, Italy, and since 1998, he has been a "Professore Incaricato" of EM fields and EM compatibility. Since November 2001, he is a Faculty Member with the Dept. of Innovation Engineering, University of Salento, Lecce, Italy, where he is Full Professor of Electromagnetic Fields. He has authored approximately 300 scientific papers. His main contributions are in the modeling of microscopic interactions of EM fields and biosystems, and in numerical methods for efficient computer-aided design (CAD) of microwave circuits and antennas. He is currently involved in bioelectromagnetics, EM energy harvesting and wireless power transmission, novel CAD tools and procedures for MW circuits, RFID, and EM high-performance computing. 